Gynäkol Rundsch 1989;29(Suppl 3):I-V

\title{
Contents, Vol. 29, Supplement 3, 1989
}

Inhalt

Vorwort

1

Die erweiterte vaginale Totalexstirpation nach Schauta-Amreich beim

Zervixkarzinom im Wandel der Zeiten

Gitsch, E.; Gitsch, G 2

Die Entwicklung der postoperativen Strahlentherapie

Kucera, $\mathrm{H} \quad 13$

Die axilläre Lymphonodektomie beim Mammakarzinom

Kubista, E 16

Blutflussmessungen der utero-plazento-fetalen Einheit

Philipp, K 21

Die konservative Behandlung der frühen Tubargravidität

Husslein, P 28

Das aktuelle Therapiekonzept beim Ovarialkarzinom

Salzer, H 35

Die Immunszintigraphie in der Onkologie am Beispiel des Ovarial-

karzinoms

Pateisky, N 40

Die Bedeutung humaner Papillomavirus-Infektionen beim Zervixkarzi

nom und seiner Vorstufen (einschliesslich anderer HPV-bedingter

genitaler Läsionen)

Czerwenka, K.F.; Schön, H.J 44

Die Entwicklung der Zyklusüberwachung in Wien

Huber,J.C 54 